\title{
Endogenous sulfur dioxide protects against isoproterenol-induced myocardial injury and increases myocardial antioxidant capacity in rats
}

\author{
Yinfang Liang ${ }^{1, *}$, Die Liu ${ }^{1, *}$, Todd Ochs ${ }^{2}$, Chaoshu Tang ${ }^{3,4}$, Stella Chen ${ }^{5}$, Suqing Zhang ${ }^{1}$, Bin Geng ${ }^{3,4}$, \\ Hongfang $\operatorname{Jin}^{1}$ and Junbao $\mathrm{Du}^{1,4}$
}

Recently, sulfur dioxide $\left(\mathrm{SO}_{2}\right)$ was discovered to be produced in the cardiovascular system and to influence important biological processes. Here, we investigated changes in endogenous $\mathrm{SO}_{2}$ /glutamic oxaloacetic transaminase (GOT) pathway in the development of isoproterenol (ISO)-induced myocardial injury in rats and the regulatory effect of $\mathrm{SO}_{2}$ on cardiac function, myocardial micro- and ultrastructure, and oxidative stress. Wistar male rats were divided into control, ISO-treated, $\mathrm{ISO}+\mathrm{SO}_{2}$, and $\mathrm{SO}_{2}$ groups. At the termination of the experiment, parameters of cardiac function and hemodynamics were measured and the micro- and ultrastructure of myocardium and stereological ultrastructure of mitochondria were analyzed. Myocardial $\mathrm{SO}_{2}$ content was detected by high-performance liquid chromatography. GOT (key enzyme for endogenous $\mathrm{SO}_{2}$ production) activity and gene (GOT1 and GOT2) expressions were measured, and superoxide dismutase (SOD), glutathione peroxidase (GSH-Px), hydrogen peroxide, and superoxide radical levels were assayed. SOD (SOD1 and SOD2) and GSH-Px (GSH-Px1) gene expressions were also detected. The results showed that $\mathrm{SO}_{2}$ donor at a dose of $85 \mathrm{mg} /(\mathrm{kg}$ day) did not impact the cardiac function and structure of rats, but exerted a subtle influence on myocardial redox status. ISO-treated rats exhibited decreased cardiac function, damaged myocardial structures, and downregulated endogenous $\mathrm{SO}_{2} / \mathrm{GOT}$ pathway. Meanwhile, myocardial oxidative stress increased, whereas antioxidative capacity downregulated. Administration of $\mathrm{SO}_{2}$ markedly improved cardiac function and ISO-induced myocardial damage by ameliorating the pathological structure of the myocardium and the mitochondria. At the same time, myocardial products of oxidative stress decreased, whereas antioxidative capacity increased. These results suggest that downregulation of the endogenous $\mathrm{SO}_{2}$ /GOT pathway is likely involved in the pathogenesis of ISO-induced myocardial injury. $\mathrm{SO}_{2}$ protects against ISO-induced myocardial injury associated with increased myocardial antioxidant capacity in rats. Laboratory Investigation (2011) 91, 12-23; doi:10.1038/labinvest.2010.156; published online 23 August 2010

KEYWORDS: antioxidant capacity; isoproterenol; myocardial injury; sulfur dioxide

Myocardial injury is a common feature in a variety of cardiac diseases. Factors proposed to be involved in its mechanism include hypoxia due to myocardial hyperactivity and coronary hypotension, calcium overload, depletion of energy reserves, and excessive production of oxygen-free radicals, resulting from the oxidative metabolism of catecholamines. The oxidative products of catecholamines affect the cardiac myocyte membrane and depress cardiac contractile activity, which are followed by damages in the mitochondria, the sarcotubular system, and contractile elements. ${ }^{1}$ Previous studies have shown isoproterenol (ISO)-induced cardiotoxicity to be related to the formation of oxygen-free radicals and sulfhydryl reactivity through a variety of oxidative products. ${ }^{2}$ Oxygen-free radicals, in turn, may increase membrane permeability by promoting lipid peroxidation, leading to the development of cardiac injury. ${ }^{3}$ However, the mechanism responsible for myocardial injury is not completely understood.

Sulfur dioxide $\left(\mathrm{SO}_{2}\right)$ was only considered as a common air pollutant in the past. Long-term exposure to high dose of $\mathrm{SO}_{2}$ has toxic effects. ${ }^{4-6}$ Interestingly, however, sulfur amino

\footnotetext{
'Department of Pediatrics, Peking University First Hospital, Beijing, PR China; ${ }^{2}$ Northwestern University, Chicago, IL, USA; ${ }^{3}$ Department of Physiology and Pathophysiology, Peking University Health Science Center, Beijing, PR China; ${ }^{4}$ Key Laboratory of Molecular Cardiovascular Diseases, Ministry of Education, Beijing, PR China and ${ }^{5}$ Department of Biochemistry and Cellular Biology, University of California, San Diego, La Jolla, CA, USA

Correspondence: Professor J Du, MD or Dr H Jin, PhD, Department of Pediatrics, Peking University First Hospital, Beijing 100034, PR China.

E-mail: junbaodu1@126.com or jinhongfang51@126.com

*These two authors contributed equally to this work. 
acid metabolism in the human body may generate $\mathrm{SO}_{2}$. As reported in literature, $\mathrm{SO}_{2}$ could be derived from various types of sulfur amino acid metabolism in the body. ${ }^{7}$ The generation of $\mathrm{SO}_{2}$ from L-cysteine occurs as follows: cysteine oxidase oxidizes L-cysteine to L-cysteinesulfinate, and the latter, by two competing pathways of decarboxylation and transamination, changes to hypotaurine and $\beta$-sulfinylpyruvate, respectively, and is finally metabolized to taurine and pyruvate. ${ }^{8}$ Through glutamic oxaloacetic transaminase (GOT), L-cysteinesulfinate is transformed into $\beta$-sulfinyl acetone acid ( $\beta$-sulfinylpyruvate), which spontaneously divides into pyruvate and $\mathrm{SO}_{2}$. In vivo, $\mathrm{SO}_{2}$ reacts with water, becoming $\mathrm{SO}_{3}^{2-}$, which in turn changes into $\mathrm{SO}_{4}^{2-}$ after being catalyzed by sulfite oxidase (Figure 1).

Ji et $a l^{9}$ used high performance liquid chromatography (HPLC) to detect the total serum concentrations of sulfite in normal subjects. Investigators identified $\mathrm{SO}_{2}$ in coronary artery, which was suggested to be an endothelium-derived hyperpolarizing factor. ${ }^{10}$ Recently, our research group found that $\mathrm{SO}_{2}$ could be endogenously generated in vascular tissues, such as aorta, pulmonary artery, mesenteric artery, tail artery and renal artery, and aorta had the highest $\mathrm{SO}_{2}$ content among the vascular tissues tested. We also found that aortic aspartate aminotransferase mRNA situated at endothelium and vascular smooth muscle cells near the endothelial layer. ${ }^{7}$

Previously, the biological role of $\mathrm{SO}_{2}$ had not been studied thoroughly. ${ }^{10}$ Zhang, from our research group, ${ }^{11}$ proved that endogenous $\mathrm{SO}_{2}$ had an important role in the regulation of myocardial ischemia-reperfusion. Recent studies revealed that exogenous $\mathrm{SO}_{2}$ has a role in the inflammatory response. ${ }^{12-14}$ Endogenous $\mathrm{SO}_{2}$ may be a biologically active molecule involved in the regulation of cardiovascular function, demonstrated by the regulatory role in the myocardial ischemia-reperfusion. ${ }^{11}$ The above findings suggested that endogenous $\mathrm{SO}_{2}$ might be an important mediator in the cardiovascular system, but it remains unclear whether and how endogenous $\mathrm{SO}_{2}$ contributes to the pathophysiological process of myocardial injury.

To evaluate the role of the $\mathrm{SO}_{2}$ /GOT pathway in the pathogenesis of myocardial injury, we attempted to observe the changes in the endogenous myocardial $\mathrm{SO}_{2}$ /GOT pathway that occurs during the development of a commonly used experimental rat model of myocardial injury, ISO-induced myocardial injury, and identify the regulatory effect of $\mathrm{SO}_{2}$ on the myocardial structure and function, and oxidative stress as well.

\section{MATERIALS AND METHODS Animal Preparation and Reagents}

Experimental protocols complied with the Animal Management Guidelines of the Ministry of Health of the People's Republic of China, and the Guide for the Care and Use of Laboratory Animals of Peking University First Hospital (Beijing, PR China).

Male Wistar rats (220-250g) were provided by the Animal Department, Health Science Center of Peking University. ISO, sodium sulfite/sodium bisulfite $\left(\mathrm{Na}_{2} \mathrm{SO}_{3} /\right.$ $\mathrm{NaHSO}_{3}$, the $\mathrm{SO}_{2}$ donor), and monobromobimane $(\mathrm{mBrB})$ were purchased from Sigma (St Louis, MO, USA); Trizol was from Gibco (BRL, Rockville, MD, USA); and deoxyribonucleotide triphosphate (dNTP), Moloney murine leukemia virus reverse transcriptase (MMLV RT), oligo$(\mathrm{dT})_{15}$, and Taq DNA polymerase were from Promega (Madison, WI, USA). Other chemicals and reagents were of analytical grade.

A total of 46 rats were randomly divided into four groups: control group $(n=16)$, ISO-treated group $(n=12)$, ISO$\mathrm{SO}_{2}$ group $(n=12)$, and $\mathrm{SO}_{2}$ group $(n=6)$. ISO-induced subacute myocardial injury was produced as described ${ }^{15}$ with

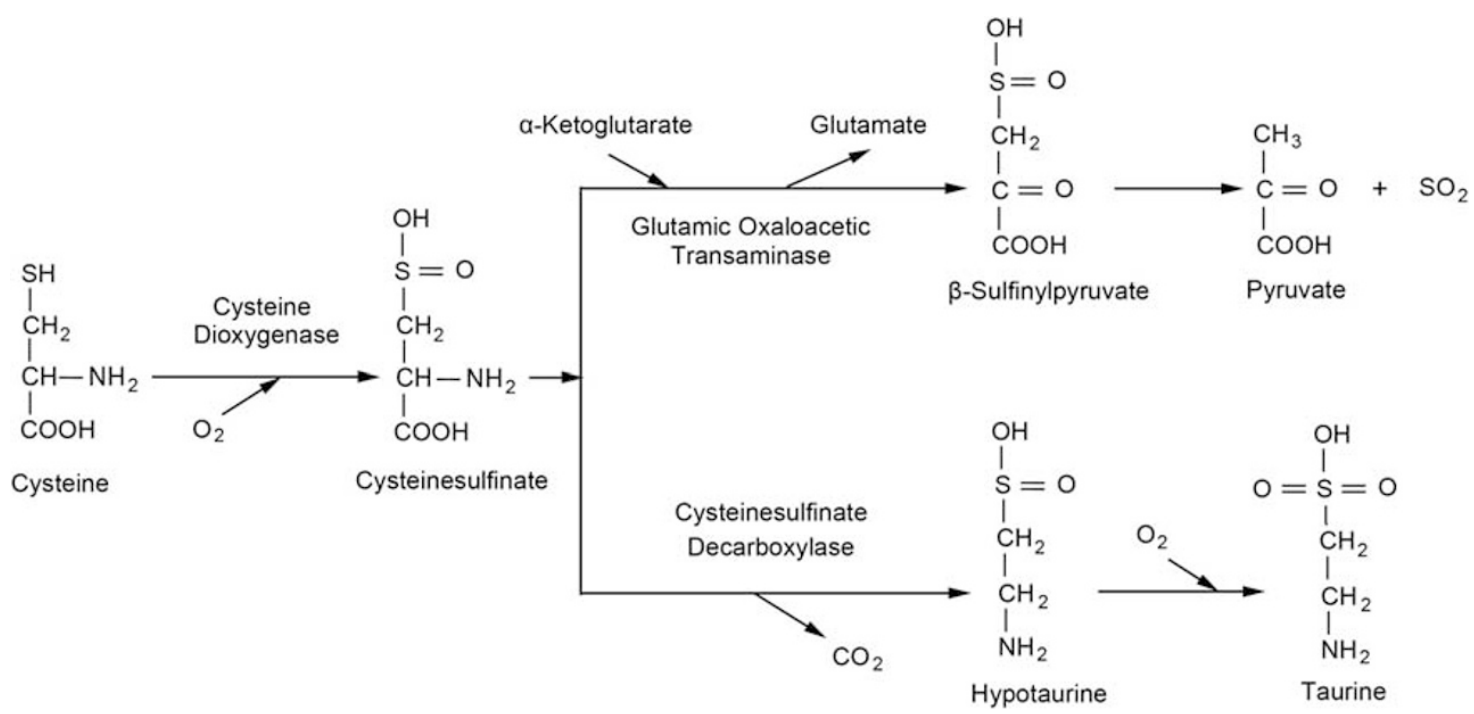

Figure 1 Sulfur amino acid metabolism pathways. Cysteine oxidase oxidizes cysteine to cysteinesulfinate, and the latter, by decarboxylation and transamination, changes to hypotaurine and sulfinylpyruvate, respectively, and finally metabolizes to taurine and pyruvate, as well as $\mathrm{SO}_{2}$. 
minor modifications. Briefly, ISO at a dose of $20 \mathrm{mg} /(\mathrm{kg}$ day $)$ was injected (subcutaneously) for 7 days in rats of the ISOtreated group. Rats were injected with $0.9 \%$ saline $(0.2 \mathrm{ml} /$ day) in the control group. Rats in the ISO $+\mathrm{SO}_{2}$ group were administered with ISO at a dose of $20 \mathrm{mg} /(\mathrm{kg}$ day) (subcutaneously) for 7 days and injected (intraperitoneally) with $\mathrm{Na}_{2} \mathrm{SO}_{3} / \mathrm{NaHSO}_{3}\left(\mathrm{SO}_{2}\right.$ donor $)$ at $85 \mathrm{mg} /(\mathrm{kg}$ day $)$ for 7 days, ${ }^{16}$ whereas rats in $\mathrm{SO}_{2}$ group were injected only with $\mathrm{Na}_{2} \mathrm{SO}_{3} /$ $\mathrm{NaHSO}_{3}$ at $85 \mathrm{mg} /(\mathrm{kg}$ day) for 7 days.

\section{Echocardiographic Examination}

Rats were anesthetized by inhalation of 3\% isoflurane and the examination was performed with a $17.5 \mathrm{MHz}$ phased-array Visual Sonics Vevo 770 Imaging System (Toronto, Canada) at the left $30^{\circ}$ decubitus position. Two-dimensional parasternal long axis views and short axis views were obtained at the papillary muscle level. Diastolic left ventricular posterior wall thickness (LVPWd) and systolic left ventricular posterior wall thickness (LVPWs) were measured. Ejection fraction (EF) and fractional shortening (FS) were then calculated. All measurements were averaged for three consecutive cardiac cycles and the examination was made by an experienced technician who was blinded to the group identity.

\section{Determination of Cardiac Function by Cardiac Catheterization}

Rats were fasted overnight but had free access to water after the last administration of the drug. At the end of the experiment, rats were anesthetized with $12 \%$ urethane $(10 \mathrm{ml} / \mathrm{kg}$, intraperitoneally). A catheter filled with heparin saline $(500 \mathrm{U} / \mathrm{ml})$ was inserted into the right carotid artery for the development of maximal left-ventricular pressure (LV $\pm \mathrm{dp} / \mathrm{dt}$ max; BL-Newcentury, Chengdu, China). Finally, the rats were killed, the hearts were removed, and the sections were formalin fixed. The rest of the rat hearts were quickly frozen in liquid nitrogen to aid detection of other indicators.

\section{Myocardial Pathological Examination}

Samples of apical ventricular muscle were fixed and dehydrated with $10 \%$ formalin. They were then embedded in paraffin and sectioned in increments of $5 \mu \mathrm{m}$. They were stained with hematoxylin and eosin ( $\mathrm{H}$ and $\mathrm{E}$ staining) and observed by optical microscopy. Fresh myocardial tissues were cut into pieces $\left(1 \mathrm{~mm}^{3}\right)$, fixed with $3 \%$ glutaraldehyde, flushed with phosphate-buffered saline (PBS), fixed with $1 \%$ perosmic acid, and dehydrated with acetone. Ultrathin sections were placed on 400-mesh grids and double stained with uranyl acetate and lead citrate. Sections were observed under transmission electron microscopy (TEM; JEM1230; Jeol, Tokyo, Japan).

\section{Stereological Analysis}

Five of the ultrathin sections from each rat were selected at random for further stereological analysis in TEM. A total of 10 microscopic fields were randomly chosen at each section and photographed. Numbers of mitochondrial profiles per picture were counted by the point counting method, as described previously. ${ }^{17}$ On the basis of this method, the number of mitochondria per picture $(N)$, mean surface area $(\bar{S})$, mean volume $(\bar{V})$, volume density $(\mathrm{Vv})$, surface density $(\mathrm{Sv})$, numerical density (Nv), and specific surface (Rsv, surface-to-volume ratio) of mitochondria were calculated following the B100 double square lattice system with the number of test points $\mathrm{Pt}=400 .^{18,19}$ All of the stereological evaluations were conducted with assistance from a Computerized Hardware-Software System (Motic Med Computer Image Analysis System 6.0; Beijing Motic Image Technology Company, Beijing, China).

\section{Determination of Tissue Sulfite Content}

Sulfite is the hydrated form of $\mathrm{SO}_{2}$ found in mammalian plasma. ${ }^{9}$ We detected the sulfite concentrations in the myocardium tissues to indirectly represent $\mathrm{SO}_{2}$ content. Sulfite determination was analyzed using HPLC with fluorescence detection (HPLC-FD; LC-20A, Shimadju, Japan). ${ }^{20}$ Briefly, $100 \mu \mathrm{l}$ of the sample was mixed with $70 \mu \mathrm{l}$ of $0.212 \mathrm{~mol} / \mathrm{l}$ sodium borohydride in $0.05 \mathrm{~mol} / \mathrm{l}$ Tris- $\mathrm{HCl}(\mathrm{pH} 8.5)$ and incubated at room temperature for $30 \mathrm{~min}$. The sample was mixed with $10 \mu \mathrm{l}$ of $70 \mathrm{mmol} / \mathrm{lmBrB}$ in acetonitrile, incubated for $10 \mathrm{~min}$ at $42^{\circ} \mathrm{C}$, and mixed with $40 \mu \mathrm{l}$ of $1.5 \mathrm{~mol} / \mathrm{l}$ perchloric acid. The protein precipitate in this mixture was removed by centrifugation at $12,400 \mathrm{~g}$ for $10 \mathrm{~min}$ at $23^{\circ} \mathrm{C}$. The supernatant was immediately neutralized by adding $10 \mu \mathrm{l}$ of $2 \mathrm{~mol} / \mathrm{l}$ Tris- $\mathrm{HCl}$ ( $\mathrm{pH} 3.0$ ) and centrifuged at $12,400 \mathrm{~g}$ for $10 \mathrm{~min}$. The neutralized supernatant was used for HPLC. The column $(4.6 \mathrm{~mm} \times 150 \mathrm{~mm} \mathrm{C18}$ reversephase column, Agilent series 1100; Agilent Technologies, Waldbronn, Karlsruhe, Germany) was first equilibrated with a buffer (methanol:acetic acid:water $=$ 5.00:0.25:94.75 by volume; $\mathrm{pH}$ 3.4). The sample loaded onto the column was resolved by a gradient of methanol for $0-5 \mathrm{~min}, 3 \%$; 5-13 min, 3-35\%; 13-30 min, 35-62\%; 30-31 min, 62-100\%; $31-39 \mathrm{~min}, 100 \%$; $39-40 \mathrm{~min}, 100-3 \%$; and $40-45 \mathrm{~min}, 3 \%$ at a flow rate of $1.0 \mathrm{ml} / \mathrm{min}$. Sulfite-bimane was measured by excitation at $392 \mathrm{~nm}$ and emission at $479 \mathrm{~nm}$. Quantification was carried out by the standardization of $\mathrm{Na}_{2} \mathrm{SO}_{3}$. Sulfite content in tissues was expressed as micromole per gram of protein.

\section{Assay of GOT Activity}

GOT activity in myocardial homogenates was determined by continuous assay and a biochemistry analyzer (Hitachi 7600, Tokyo, Japan). GOT activity in the myocardium was expressed as units per milligram of protein.

\section{Determination of GOT1, GOT2, Superoxide Dismutase 1, Superoxide Dismutase 2 and Glutathione Peroxidase mRNAs in Tissues}

Total RNA in tissues was extracted using Trizol reagent and reverse transcribed by oligo- $(\mathrm{dT})_{18}$ primer and MMLV RT. 
Primers and probes used are listed in Table 1. Quantitative real-time PCR was carried out on an ABI PRISM 7300 instrument. The PCR mixture contained $5 \mu \mathrm{l}$ of $10 \times$ PCR buffer, $5 \mu \mathrm{l}$ of cDNA template or standard DNA, $4 \mu \mathrm{l}$ of $2.5 \mathrm{mM}$ per dNTPs, $5 \mathrm{U}$ of Taq DNA polymerase, $1 \mu \mathrm{l}$ of ROX (catalog number 12223-012; Invitrogen, Carlsbad, CA, USA), $15 \mathrm{pmol}$ each of forward and reverse primers, and $10 \mathrm{pmol}$ of TaqMan probe in a total volume of $50 \mu \mathrm{l}$. Samples and standard DNAs were determined in duplicate. The PCR condition was predenatured at $95^{\circ} \mathrm{C}$ for $5 \mathrm{~min}$, then $95^{\circ} \mathrm{C}$ for $15 \mathrm{~s}$, and $60^{\circ} \mathrm{C}$ for $1 \mathrm{~min}$ for 40 cycles. The amount of $\beta$-actin cDNA in the sample was used to calibrate the sample amount used for determination.

\section{Measurement of Oxidants and Antioxidative Enzymes in Myocardium Tissue}

Myocardium tissue was rinsed with saline and resected. A ninefold volume of PBS was added, and the mixture was ground gently at $4^{\circ} \mathrm{C}$. Concentrations of superoxide dismutase (SOD) and glutathione peroxidase (GSH-Px) in myocardial homogenates were determined according to the xanthine oxidase method and 5,5'-dithiobis-2-nitrobenzoic acid method provided by standard assay kits (Nanking Jiancheng Biology Company, Nanjing, China), as described previously. ${ }^{21}$ Superoxide radical $\left(\mathrm{O}_{2}^{\bullet-}\right)$ production was assessed from myocardial segments by the lucigenin chemiluminescence $(5 \mu \mathrm{mol} / \mathrm{l})$ method, as we previously described. ${ }^{22-25}$ Hydrogen peroxide $\left(\mathrm{H}_{2} \mathrm{O}_{2}\right)$ production in myocardial homogenate was evaluated according to the modified protocols of Miura et al. ${ }^{26}$

\section{Statistics}

Results were expressed as mean \pm s.d. All data were analyzed with SPSS 11.5 (SPSS, Incorporated, Chicago, IL, USA), and analysis of variance (ANOVA) followed by post-hoc analysis (Newman-Keuls test) was used to compare differences among groups. $P<0.05$ was considered significant.

Table 1 Primers and TaqMan probes used in quantitative real-time PCR for the measurement of glutamic oxaloacetic transaminase 1, glutamic oxaloacetic transaminase 2 , superoxide dismutase 1 , superoxide dismutase 2 , glutathione peroxidase 1 and $\beta$-actin cDNAs in rat

\begin{tabular}{|c|c|c|c|}
\hline cDNA & Oligonucleotide & Sequence & Product size (bp) \\
\hline & Forward primer & 5'-CCAGGGAGCTCGGATCGT-3' & 79 \\
\hline & TaqMan probe & $5^{\prime}$-CCACCACCCTCTCCAACCCTGA-3' & \\
\hline \multirow[t]{3}{*}{ Glutamic oxaloacetic transaminase 2} & Reverse primer & 5'-GTTCCCCAGGATGGTTGG-3' & \\
\hline & TaqMan probe & 5'-TTAAGTTCAGCCGAGATGTCTITC-3' & \\
\hline & Forward primer & 5'-AAAGGACGGTGTGGCCAAT-3' & 121 \\
\hline Superoxide dismutase 1 & Reverse primer & 5'-TCCACCTTGCCCAAGTCAT-3' & \\
\hline \multirow[t]{3}{*}{ Superoxide dismutase 2} & Reverse primer & 5'-GGCTCAGGTTTGTCCAGAAAAT-3' & \\
\hline & TaqMan probe & 5'-AGGCGCTGGCCAAGGGAGAT-3' & \\
\hline & Forward primer & 5'-CGTGCAATCAGTTCGGACAT-3' & 131 \\
\hline \multirow[t]{3}{*}{ Glutathione peroxidase 1} & Reverse primer & 5'-CTCACCATTCACCTCGCACTT-3' & \\
\hline & TaqMan probe & 5'-AATGAAGAGATTCTGAATTCCCTCAAGT-3' & \\
\hline & Forward primer & 5'-ACCCGCGAGTACAACCTTCTT-3' & 80 \\
\hline
\end{tabular}

TaqMan probe labeled with FAM at the $5^{\prime}$-end and TAMRA at the $3^{\prime}$-end 


\section{RESULTS}

\section{Parameters of Cardiac Function Evaluated by Echocardiography}

LVPWd and LVPWs significantly increased by 30.7 and $21.7 \%$ in rats of ISO group compared with those of the control group $(P<0.01)$, whereas they significantly decreased by 14.7 and $9.1 \%$ in $\mathrm{ISO}+\mathrm{SO}_{2}$ group compared with those of the ISO group $(P<0.05)$. EF and FS significantly decreased by 23.6 and $32.4 \%$ in rats of ISO group compared with those of the control group $(P<0.01)$, whereas they significantly increased by 27.5 and $30.6 \%$ in $\mathrm{ISO}+\mathrm{SO}_{2}$ group compared those of the ISO group $(P<0.01)$. The four parameters of cardiac function, LVPWd, LVPWs, EF, and FS, exhibited no variation in $\mathrm{SO}_{2}$-treated rats compared with those of controls (all, $P>0.05$; Table 2).

\section{Parameters of Cardiac Function Evaluated by Cardiac Catheterization}

Compared with control rats, ISO-treated rats showed lowered $\mathrm{LV} \pm \mathrm{dp} / \mathrm{dt}$ max values, whose $\mathrm{LV}+\mathrm{dp} / \mathrm{dt} \max$ and $\mathrm{LV}-\mathrm{dp} / \mathrm{dt}$ $\max$ values decreased by 22.3 and $39.0 \%$, respectively $(P<0.01)$; after administration of exogenous $\mathrm{SO}_{2}$, their $\mathrm{LV}+\mathrm{dp} / \mathrm{dt} \max$ and $\mathrm{LV}-\mathrm{dp} / \mathrm{dt}$ max values increased by 9.6 and $22.6 \%$, respectively $(P<0.01$ and $P<0.05)$. However, there was no significant difference in $\mathrm{LV}+\mathrm{dp} / \mathrm{dt} \max$ and $\mathrm{LV}-\mathrm{dp} / \mathrm{dt}$ max values between $\mathrm{SO}_{2}$-treated rats and controls (both, $P>0.05$; Figure 2).

\section{Myocardial Pathological Changes}

$\mathrm{H}$ and $\mathrm{E}$ staining in the control group showed that cardiomyocytes were arranged in rows, the fiber structure of cardiomyocytes was clear, there was no damage to cardiac fibers, and the cytoplasm was in uniform (Figure 3a). Subcutaneous administration of ISO caused necrosis of myocardial cells, dissolution of cardiomyocytes, and infiltration of monocytes and other inflammatory cells (Figure 3b). After intraperitoneal administration of $\mathrm{SO}_{2}$, necrotic foci, proliferation of fiber granulation tissue, and infiltration of

Table 2 Echocardiographic parameters in different groups (mean \pm s.d.)

\begin{tabular}{|c|c|c|c|c|}
\hline & EF (\%) & FS (\%) & $\begin{array}{l}\text { LVPWs } \\
(\mathrm{mm})\end{array}$ & $\begin{array}{l}\text { LVPWd } \\
(\mathrm{mm})\end{array}$ \\
\hline Control $(n=6)$ & $78.2 \pm 3.04$ & $48.6 \pm 3.00$ & $2.53 \pm 0.25$ & $1.66 \pm 0.19$ \\
\hline ISO group $(n=6)$ & $59.7 \pm 3.96^{\# \#}$ & $32.8 \pm 2.73^{\# \#}$ & $3.08 \pm 0.33^{\# \#}$ & $2.17 \pm 0.47^{\# \#}$ \\
\hline $\begin{array}{l}\mathrm{ISO}+\mathrm{SO}_{2} \text { group } \\
(n=6)\end{array}$ & $76.2 \pm 6.19^{* *}$ & $47.3 \pm 5.95^{* *}$ & $2.80 \pm 0.80^{*}$ & $1.85 \pm 0.15^{*}$ \\
\hline $\mathrm{SO}_{2}$ group $(n=6)$ & $74.5 \pm 2.92$ & $44.8 \pm 2.70$ & $2.54 \pm 0.30$ & $1.56 \pm 0.24$ \\
\hline
\end{tabular}

Abbreviations: EF, ejection fraction; FS, fractional shortening; LVPWd, diastolic left ventricular posterior wall thickness; LVPWs, systolic left ventricular posterior wall thickness.

${ }^{\# \#} P<0.01$ vs control; ${ }^{*} P<0.01$ vs ISO group; and ${ }^{*} P<0.05$ vs ISO group. noninflammatory cells were seen (Figure 3c). No damage was observed in myocardial structure by $\mathrm{H}$ and $\mathrm{E}$ staining in $\mathrm{SO}_{2}$ group (Figure 3d). Under TEM, samples from rats in the control group showed myocardial fibers that were ordered, and that the sarcoplasmic reticulum (SR) and $\mathrm{T}$ tubular structure of mitochondria were clearly observed; the sarcolemma was integrated, and small vascular endothelial cells and the substrate were not damaged (Figure 4a). However, samples from rats in the ISO group showed a focal myocardial fiber structure that was disordered, cardiomyocytes with nuclear condensation, large vacuoles within the cytoplasm, newly-formed vacuoles, and mitochondria that were swollen and whose crests was broken and dissolved. The structure of the rest of the muscle fibers was better. SR and $\mathrm{T}$ tubules were in a high degree of expansion, the lamellar body could be seen, and fewer glycogen particles existed; mitochondrial structure and most of the sarcolemma were maintained. Small vascular endothelial cells and substrates showed no obvious abnormalities (Figure $4 \mathrm{~b}$ ). However with treatment of $\mathrm{SO}_{2}$ donor in $\mathrm{ISO}+\mathrm{SO}_{2}$ group, myocardial fibers were clear, the structure of some mitochondria was clear, but still some mitochondria were mildly swollen. SR and $\mathrm{T}$ tubules showed moderate expansion, small amounts of lipid were seen, most of the sarcolemma was integrated, and the structure of small vascular endothelial cells and the substrate were normal (Figure $4 \mathrm{c}$ ). In rats treated only with $\mathrm{SO}_{2}$, the myocardial fibers were normally arranged and the mitochondria were not swollen (Figure 4d).

Stereological analysis of cardiomyocytic mitochondria among the samples from three groups of rats showed a $16 \%$ increase in $\bar{S}, 44 \%$ in $\bar{V}$, and $23 \%$ in $\mathrm{Vv}$ of ISO-treated rats when compared with respective control values (all, $P<0.01$ ). Mitochondrial $\mathrm{N}, \mathrm{Nv}$, and Rsv in samples from rats of the ISO-treated group were reduced by $14 \%, 17 \%$, and $19 \%$ (all, $P<0.01$ ) when compared with respective control rats. Administration of $\mathrm{SO}_{2}$ donor, however, markedly attenuated

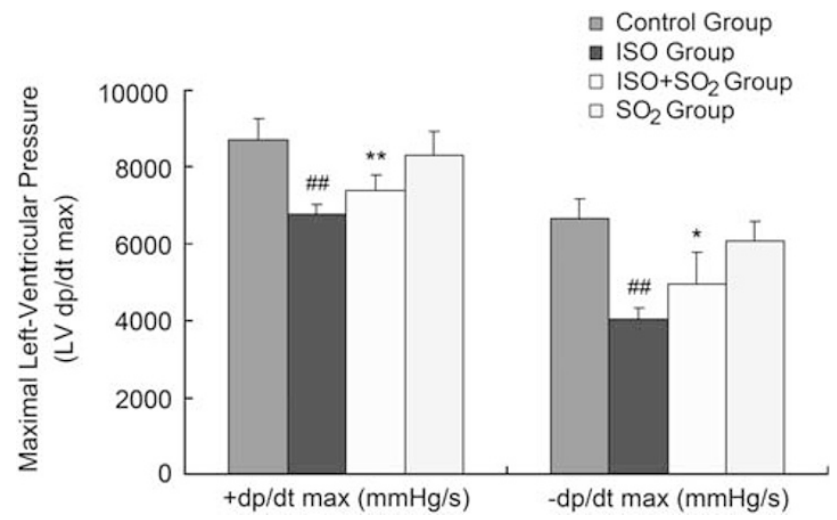

Figure 2 Maximal left-ventricular pressure ( $L V \pm d p / d t$ max) in rats of different groups. A catheter filled with heparin saline $(500 \mathrm{U} / \mathrm{ml})$ was inserted into the right carotid artery for detecting the development of $\mathrm{LV} \pm \mathrm{dp} / \mathrm{dt}$ max. ${ }^{\# \#} P<0.01$ vs control group; ${ }^{* * P}<0.01$ vs ISO group; and ${ }^{\star} P<0.05$ vs ISO group. 

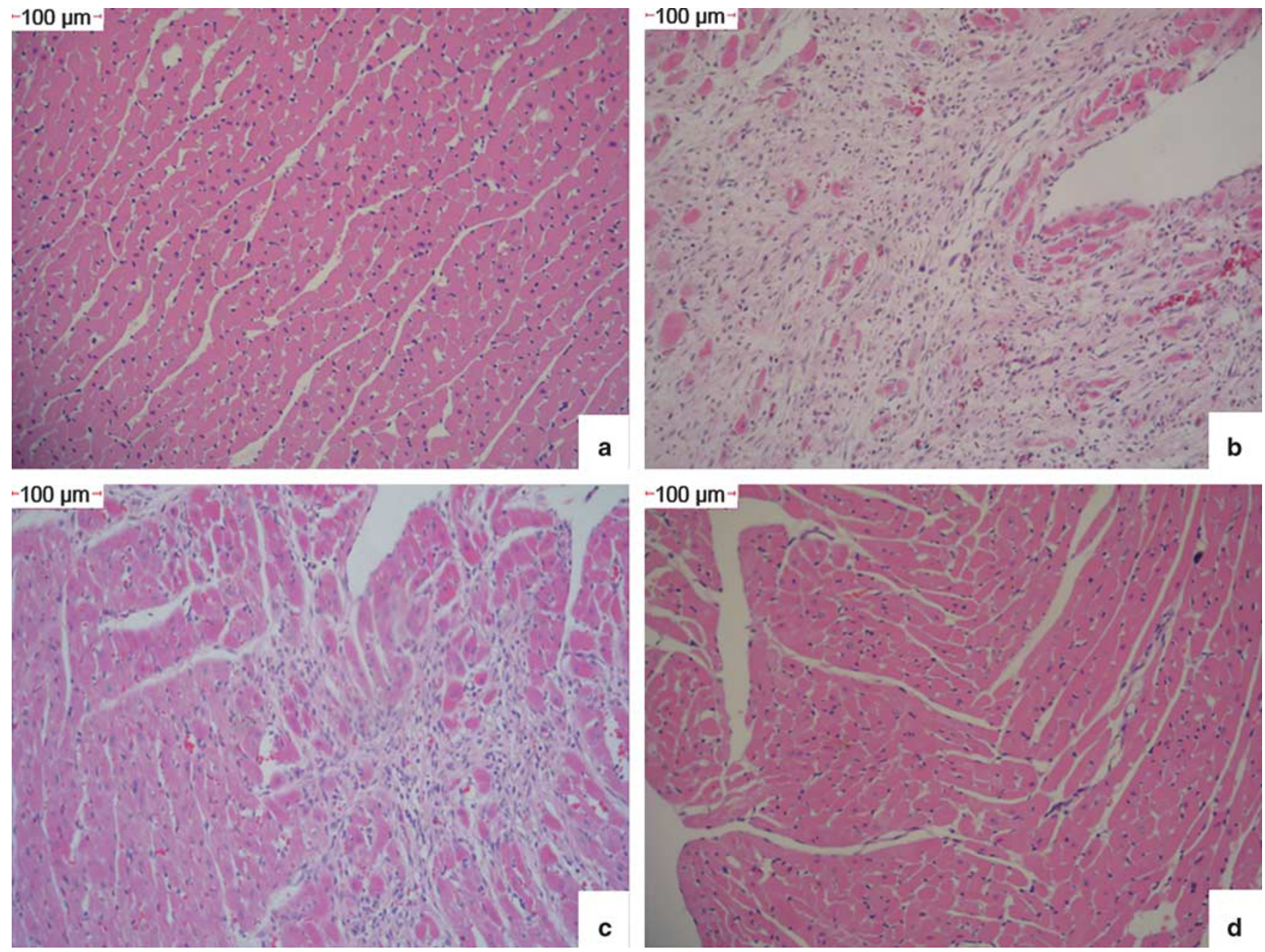

Figure 3 Microstructural changes in cardiomyocytes. $\mathrm{H}$ and $\mathrm{E}$ staining in rats showed that in controls (a), the microstructure of cardiomyocytes was normal; in ISO group (b), myocardial structure was damaged. Necrosis of myocardial cells, dissolution of myocardial cells, and infiltration of monocytes and other inflammatory cells were present; whereas in $\mathrm{ISO}+\mathrm{SO}_{2}$ group (c), the damage of myocardial structure was improved; and in $\mathrm{SO}_{2}$ group (d), the damage of myocardial structure was not observed.

ISO-induced mitochondrial swelling and deformation, as exhibited by a significant reduction in $\bar{S}, \bar{V}$, and $\mathrm{Vv}$, and a marked increase in $\mathrm{N}, \mathrm{Nv}$, and Rsv of mitochondria $(\bar{S}, \bar{V}$, and $\mathrm{Vv}$ decreased by 13,30 , and $21 \%$, all, $P<0.01 ; \mathrm{N}, \mathrm{Nv}$, and Rsv increased by 12,15 , and $24 \%$, all, $P<0.01$ ) when compared with ISO-treated rats, respectively. There was no significant difference in stereological parameters of cardiomyocytic mitochondria between the controls and $\mathrm{SO}_{2}$-treated rats (all, $P>0.05$; Figure 5).

\section{Myocardial Sulfite Level}

Sulfite content in the myocardium markedly increased by $61.5 \%$ in rats of $\mathrm{SO}_{2}$ group compared with that of control rats $(P<0.05)$. However, sulfite concentration in rat myocardial homogenates after ISO administration decreased significantly by $41.9 \%(P<0.01)$, whereas after administration of a $\mathrm{SO}_{2}$ donor, sulfite content in the myocardium of rats increased by $66.0 \%$ compared with ISO-treated rats (Figure 6a).

\section{Myocardial GOT Activity and GOT1 and GOT2 mRNA Expressions}

Compared with those of control rats, myocardial GOT activity reduced by $73.9 \%$ in ISO-treated rats and by $88.3 \%$ in $\mathrm{SO}_{2}$ group (both, $P<0.01$; Figure $6 \mathrm{~b}$ ). The GOT1 mRNA/ $\beta$ actin mRNA ratio in ISO-treated rat myocardium decreased by $63.4 \%(P<0.01)$, whereas the GOT2 $\mathrm{mRNA} / \beta$-actin mRNA ratio of myocardium of ISO-treated rats fell by $50.0 \%$ $(P<0.01$, Figure 6c $)$. The myocardial GOT1 mRNA and GOT2 mRNA did not change in $\mathrm{SO}_{2}$ group compared with controls (both, $P>0.05$; Figure $6 \mathrm{c}$ ). After administration of $\mathrm{SO}_{2}$, however, GOT activity, GOT1 mRNA, and GOT2 mRNA remained unchanged compared with those of ISO-treated rats (all, $P>0.05$; Figure $6 \mathrm{~b}$ and $\mathrm{c}$ ). 

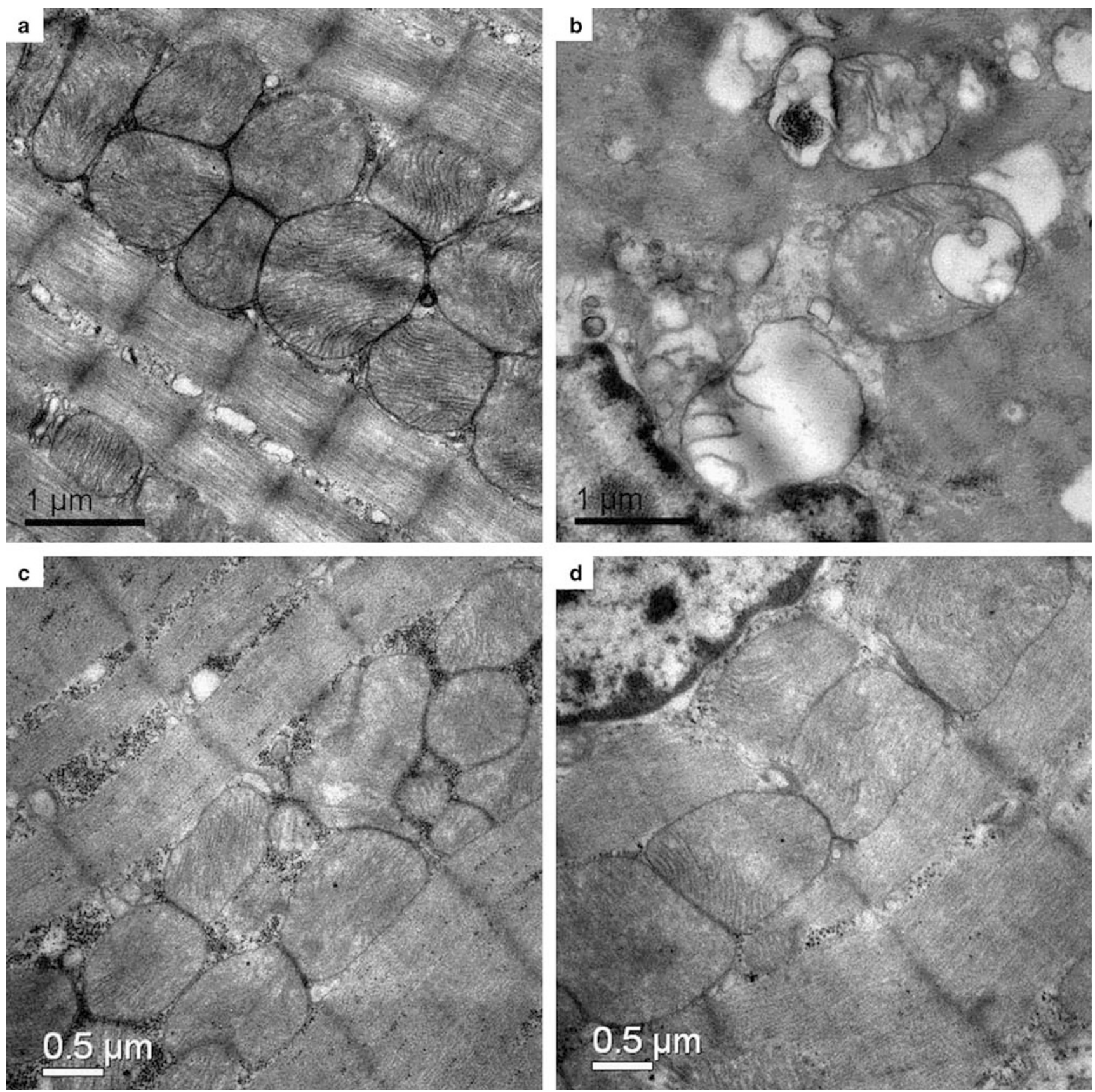

Figure 4 The ultrastructural changes in cardiomyocytes. Under TEM, it was observed that in rats in control group (a), structure of cardiomyocytes was clearly observed; in ISO group (b), myocardial fiber structure was disordered and mitochondria was swollen; whereas in $\mathrm{ISO}_{+} \mathrm{SO}_{2}$ group $(\mathbf{c})$, part of mitochondria was mildly swollen, and SR and T tubules showed moderate expansion; and in $\mathrm{SO}_{2}$ group (d), the ultrastructure of cardiomyocytes is similar to the controls.

Influence of $\mathrm{SO}_{2}$ on Oxidative and Antioxidative Balance in ISO-Induced Myocardial Injury

At 7 days after subcutaneous administration of ISO, oxidant production (that is, $\mathrm{H}_{2} \mathrm{O}_{2}$ and $\mathrm{O}_{2}^{--}$) clearly increased $(P<0.01$ and $P<0.05$, respectively) compared with those of myocardial homogenates of control rats, but the activity of the antioxidative enzymes SOD and GSH-Px reduced significantly (all, $P<0.01$ ), and SOD1, SOD2, and GSH-Px1
mRNAs downregulated by $57.1 \%, 82.3$, and $28.6 \%$, respectively (all, $P<0.01$; Figure 7 ). Meanwhile, after the intraperitoneal administration of a $\mathrm{SO}_{2}$ donor, the activity of $\mathrm{SOD}$ and GSH of myocardial homogenates of ISO $+\mathrm{SO}_{2}$-treated rats increased by 6.7 and $10.2 \%(P<0.05$ and $P<0.01$, respectively) and the mRNAs of SOD2 and GSH-Px1 in myocardial tissues upregulated by 60.0 and $28.9 \%$, respectively (both, $P<0.05$ ), whereas $\mathrm{H}_{2} \mathrm{O}_{2}$ and $\mathrm{O}_{2}^{\bullet-}$ decreased by 

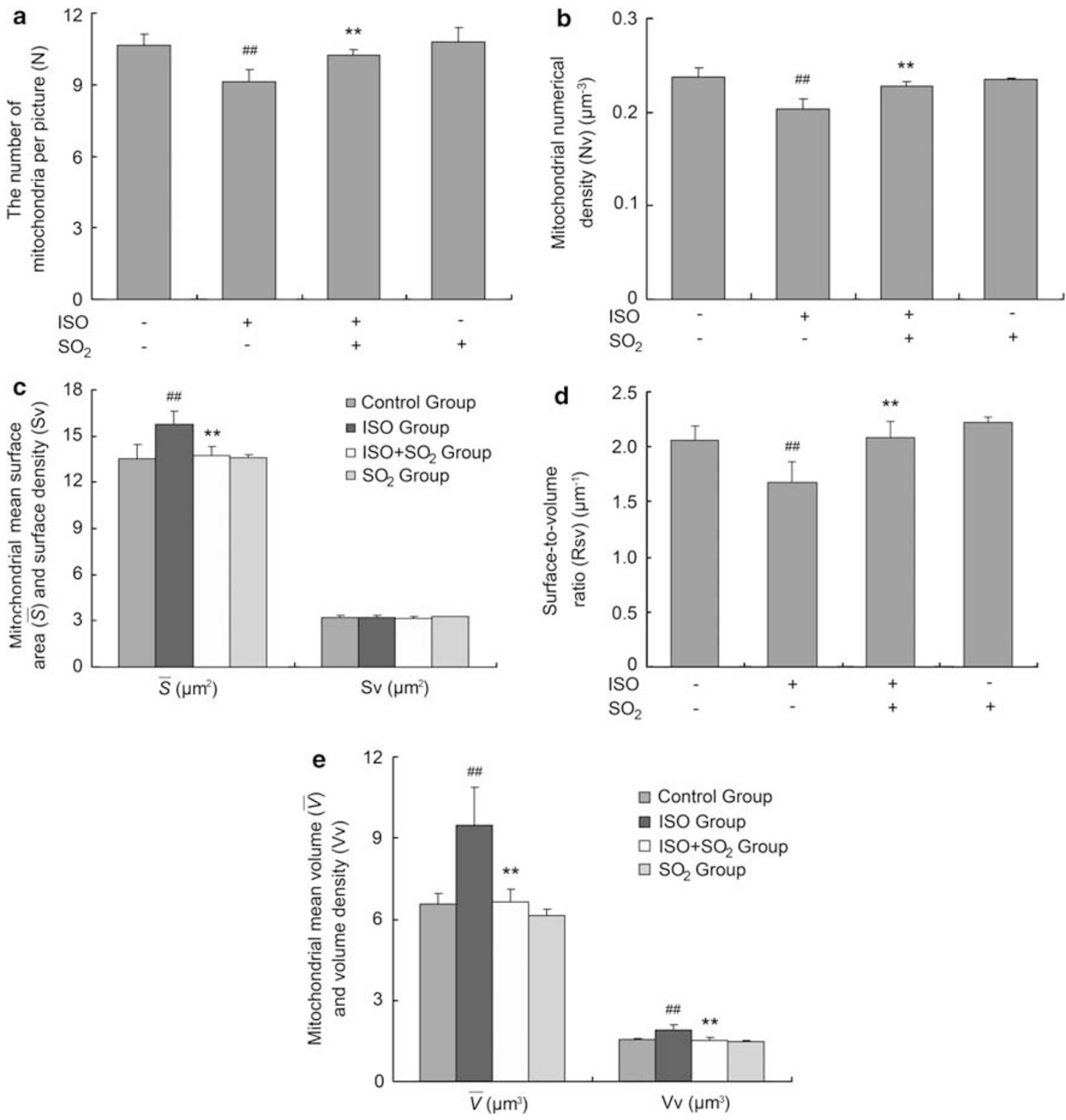

Figure 5 Stereological analysis of myocardial mitochondria using TEM. A total of 10 microscopic fields were randomly chosen at each section and photographed. (a) Mitochondrial number was reduced when rats were treated with ISO compared with controls. However, it was increased in rats of $\mathrm{ISO}+\mathrm{SO}_{2}$ group, as compared with ISO-treated rats. There was no difference between $\mathrm{SO}_{2}$ group and control group. $\mathrm{N}$ stands for the number of mitochondria per picture, ISO stands for isoproterenol, and $\mathrm{SO}_{2}$ stands for sulfur dioxide. (b) Mitochondrial numerical density was reduced when rats were treated with ISO, as compared with controls, whereas it was increased in ISO $+\mathrm{SO}_{2}$ group, as compared with ISO-treated rats. $\mathrm{Nv}$ of $\mathrm{SO}_{2}$-treated group was similar to controls. Nv stands for numerical density. (c) An increase in average surface area was found in ISO-treated rats compared with that of controls. A significant reduction in mitochondrial $\bar{S}$ was noted in rats of $\mathrm{ISO}+\mathrm{SO}_{2}$ group compared with ISO-treated rats. The disparities between the control group and $\mathrm{SO}_{2}$ group were not detected. There was no significant difference in mitochondrial Sv among rats of different groups. $\bar{S}$ stands for average surface area and Sv surface density. (d) Mitochondrial surface-to-volume ratio was reduced when rats were treated with ISO compared with controls and it was increased in rats of $\mathrm{ISO}+\mathrm{SO}_{2}$ group compared with ISO-treated rats, whereas it showed no changes in $\mathrm{SO}_{2}$ group compared with controls. Rsv stands for surface-tovolume ratio. (e) Increases in mitochondrial average volume and volume density were found in ISO-treated rats compared with those of controls; meanwhile, significant reduction in mitochondrial average volume and volume density was noted in rats of $\mathrm{ISO}_{2} \mathrm{SO}_{2}$ group compared with $\mathrm{ISO}$-treated rats, and there was no significant variation between $\mathrm{SO}_{2}$-treated and control groups. $\bar{V}$ stands for average volume and $\mathrm{Vv}$ for volume density. ${ }^{\# \#} P<0.01$ vs control group; and ${ }^{* *} P<0.01$ vs ISO group.

14.7 and $47.6 \%$, respectively, as compared with those of ISO rats $(P<0.05$; Figure 7$)$. However, mRNA of SOD1 did not change in rats of the ISO $+\mathrm{SO}_{2}$ group compared with ISOtreated rats $(P>0.05$; Figure 7$)$. The content of $\mathrm{H}_{2} \mathrm{O}_{2}$ and
$\mathrm{O}_{2}^{\bullet-}$ in rat myocardial homogenates showed no statistical variation between control group and $\mathrm{SO}_{2}$-treated group $(P>0.05)$, whereas in $\mathrm{SO}_{2}$-treated group, the activity of the antioxidative enzymes SOD1 and GSH-Px decreased 

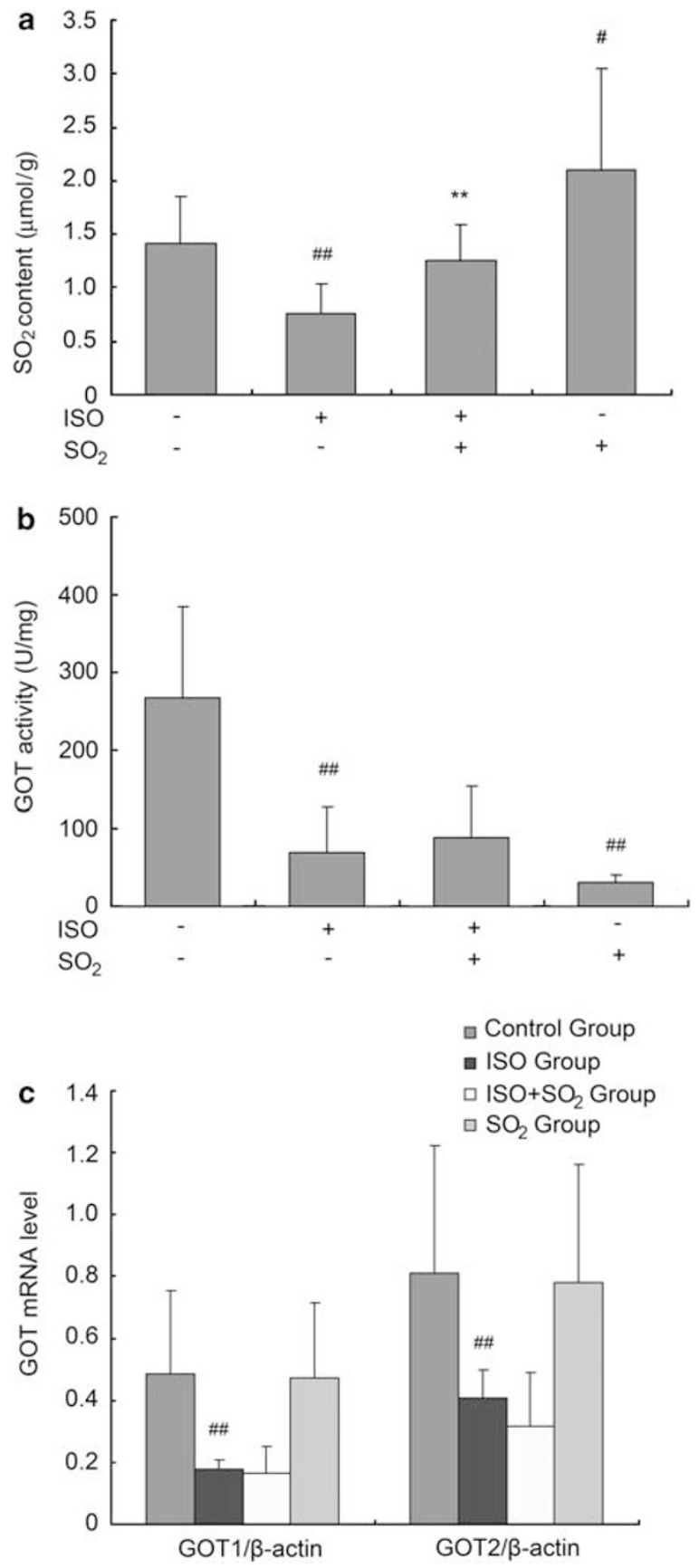

Figure 6 The changes in sulfur dioxide $\left(\mathrm{SO}_{2}\right)$ /glutamic oxaloacetic transaminase (GOT) pathway in myocardial tissue of isoproterenol (ISO)induced rats. In ISO-treated rats, myocardial sulfite content decreased compared with the controls, but it increased significantly in rats of $\mathrm{ISO}+\mathrm{SO}_{2}$ group compared with that of ISO-treated rats, and in $\mathrm{SO}_{2}$ group it was markedly higher than that of controls (a). Myocardial GOT activity reduced in both ISO group and $\mathrm{SO}_{2}$ group compared with that of control rats, but it did not change in ISO $+\mathrm{SO}_{2}$ group compared with ISO group (b). GOT1 and GOT2 gene expressions downregulated in ISO-treated rats compared with control rats, but they did not differ between $\mathrm{ISO}+\mathrm{SO}_{2}$ group and ISO group, and between $\mathrm{SO}_{2}$ group and control group (c). ${ }^{\# \#} P<0.01$ vs control group; ${ }^{\#} P<0.05$, vs control group; and ${ }^{* *} P<0.01$ vs ISO group. significantly $(P<0.01$ and $P<0.05$, respectively) accompanied by $S O D 1$ and GSH-Px1 mRNA downregulation (all, $P<0.01$, Figure 7).

\section{Discussion}

Supramaximal doses of the $\beta$-adrenergic agonist ISO induced acute myocardial necrosis and interstitial fibrosis., ${ }^{1,15,27-30}$ Histopathological lesions produced by ISO in rats resembled those from myocardial infarction in animal models of atherosclerosis. ${ }^{1}$ Grimm et $a l^{31}$ showed that supramaximal dosages of ISO led to heart failure, characterized by increases in end-diastolic volume, end-diastolic pressure, leftventricular wall thickness, and myocardial deposition of fibronectin and laminin. In this study, we used a rat model of ISO-induced myocardial injury to examine the possible role of endogenous $\mathrm{SO}_{2}$ in the development of myocardial injury.

To examine whether $\mathrm{SO}_{2}$ donor at a dose of $85 \mathrm{mg} /(\mathrm{kg}$ day) impacted the cardiac physiological status of rats, we investigated the cardiac function and structure between normal rats and $\mathrm{SO}_{2}$-treated rats. In $\mathrm{SO}_{2}$ group, the sulfite concentration in myocardial homogenates was markedly higher than that of the controls, whereas the cardiac function and structure remained unchanged, suggesting that the dose of $\mathrm{SO}_{2}$ donor we used in this study might not influence the cardiac function and structure of normal rats, although it led to a marked increase in $\mathrm{SO}_{2}$ concentration in myocardium (increased by $61.5 \%$ ) as compared with controls. The increase in myocardial concentration of $\mathrm{SO}_{2}$ led to an attenuated myocardial antioxidant capacity demonstrated by the decrease in both mRNA expression and enzyme activity of SOD and GSH-Px. However, the release of free oxygen radicals was not altered. Hence, this impact on myocardial redox status did not reach the extent to which the myocardial injury occurred.

After the treatment of ISO, myocardial contraction and diastolic function were significantly lowered, as demonstrated by decreased $+\mathrm{dp} / \mathrm{dt} \max$ and $-\mathrm{dp} / \mathrm{dt} \max$, increased LVPWd and LVPWs, and decreased EF and FS. In addition, in ISO-treated rats, $\mathrm{H}$ and $\mathrm{E}$ staining showed damaged myocardial structure. TEM revealed that the ultrastructures of cardiomyocytes were seriously destructed. The observations stated were typical of myocardial ischemic necrosis. Quantitative analysis of mitochondrial morphology indicated that the myocardial mitochondria of ISO-treated rats became swollen and necrotic. This was correlated with the alterations of mitochondria observed under TEM. The above facts demonstrated that a myocardial ischemic model was created.

Interestingly, in ISO-treated rats, myocardial sulfite content was lower than that of the control group. It has been reported that GOT is a key enzyme in controlling endogenous production of $\mathrm{SO}_{2}{ }^{32,33}$ Therefore, to investigate why the myocardial content of sulfite decreased in the ISO-treated rats, we examined the GOT activity in myocardium and 

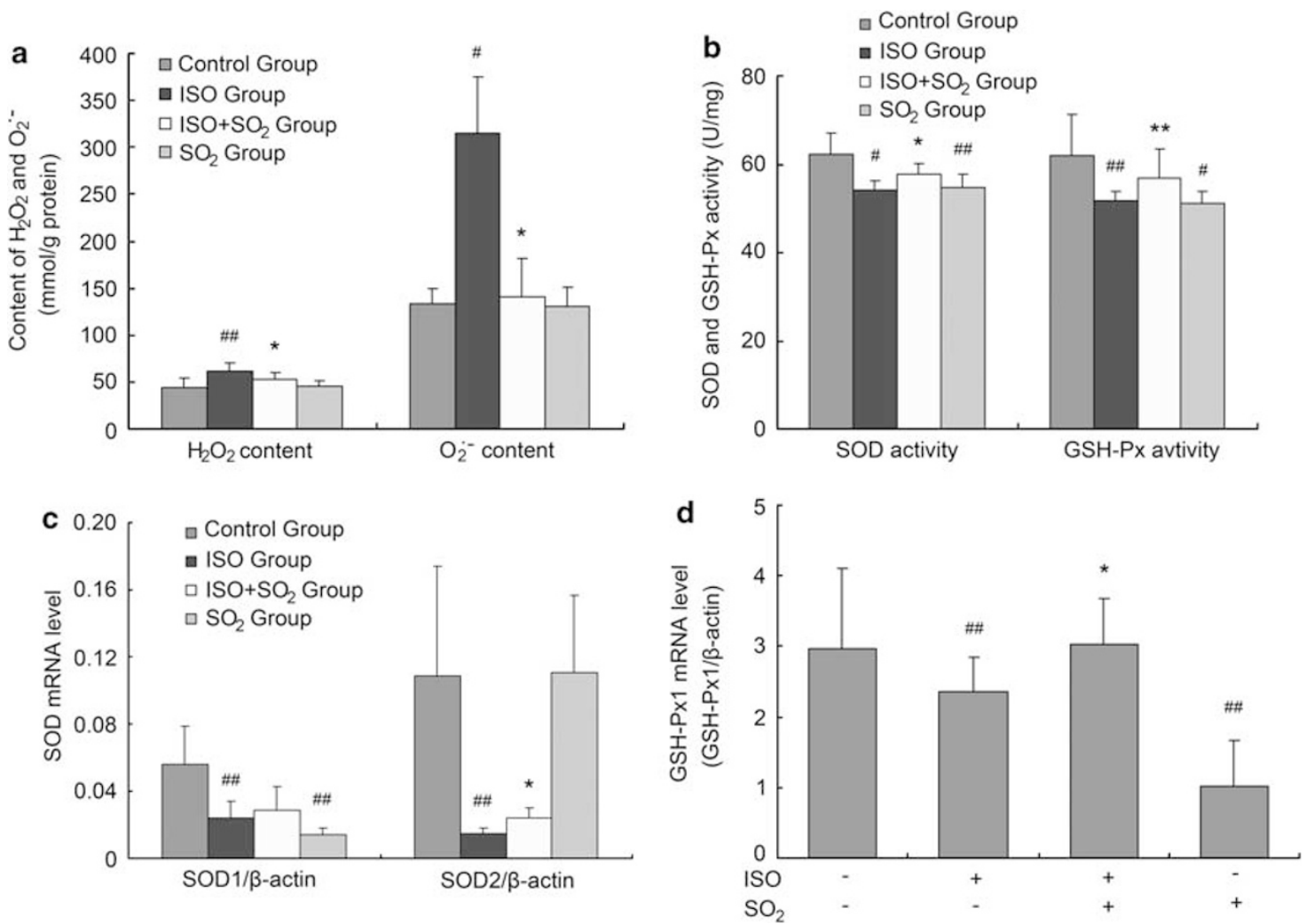

Figure 7 Measurement of oxidative stress and antioxidative capacities in myocardium tissue. In ISO group, myocardial homogenate $\mathrm{H}_{2} \mathrm{O}_{2}$ and $\mathrm{O}_{2}^{\bullet-}$ increased, compared with those of control rats. Meanwhile, in ISO $+\mathrm{SO}_{2}$ group, $\mathrm{H}_{2} \mathrm{O}_{2}$ and $\mathrm{O}_{2}^{\bullet-}$ decreased markedly, as compared with those of ISO rats. There was no difference between $\mathrm{SO}_{2}$-treated group and the control group (a). The activity of myocardial homogenate SOD and GSH-Px decreased significantly in ISO-treated rats compared with controls, as well as in $\mathrm{SO}_{2}$ group compared with controls, but they increased in $\mathrm{ISO}_{+} \mathrm{SO}_{2} \mathrm{group} \mathrm{compared}$ with those of ISO group (b). Both SOD1 and SOD2 mRNAs downregulated significantly in ISO-treated rats compared with controls, whereas SOD2 mRNAs upregulated in $\mathrm{ISO}+\mathrm{SO}_{2}$ group compared with ISO group and SOD1 mRNAs decreased in $\mathrm{SO}_{2}$ group compared with controls (c). GSH-Px1 mRNAs downregulated significantly in both ISO-treated rats and $\mathrm{SO}_{2}$-treated rats compared with controls, but upregulated in ISO $+\mathrm{SO}_{2}$ group compared with that of ISO group (d). ${ }^{\# \#} P<0.01$ vs control group; ${ }^{\#} P<0.05$ vs control group; ${ }^{* * P}<0.01$ vs ISO group; and ${ }^{*} P<0.05$ vs ISO group.

found that it also decreased significantly. Moreover, mRNAs of GOT1 and GOT2 were significantly lower than those in the control group. It was indicated that the endogenous $\mathrm{SO}_{2} / \mathrm{GOT}$ pathway was downregulated in myocardial injury induced by ISO.

To explore whether the downregulation of the $\mathrm{SO}_{2} / \mathrm{GOT}$ pathway is involved in the mechanisms responsible for ISOinduced myocardial damage, we treated ISO-injected rats with exogenous $\mathrm{SO}_{2}$. By adding exogenous $\mathrm{SO}_{2}$, sulfite content of the myocardium was significantly increased, and more interestingly, cardiac function significantly improved. In addition, myocardial pathological changes were obviously alleviated. Stereological analysis of myocardiocytic mitochondria showed an improvement from the mitochondrial injury. These results are consistent with the alleviation of morphological mitochondrial injury. The data suggest that downregulation of the endogenous $\mathrm{SO}_{2}$ /GOT pathway probably participated in the mechanisms responsible for myocardial damage induced by ISO.

Until now, the mechanisms by which $\mathrm{SO}_{2}$ regulates ISO-induced myocardial damage have been unclear. Previous studies indicated that large doses of ISO induced severe oxidative stress. ${ }^{1,34}$ Meszaros et $a l^{35}$ showed that administration of supramaximal dosages of ISO increased enddiastolic volume and end-diastolic pressure, contributing to ventricular dysfunctions, which possibly resulted from the auto-oxidation of catecholamines that stimulated oxidative stress via reactive mediators, leading to cardiotoxicity. ${ }^{36}$ More interestingly, the ventricular dysfunction, as mentioned above, could be significantly prevented by antioxidants. ${ }^{35,37}$ An increasing body of evidence suggests that free radicals, including reactive oxygen species and reactive nitrogen species, have long been recognized to act as the major mediators of cardiac injury, and antioxidants and free radical scavengers have been shown to minimize cardiac injury, which, to some extent, verifies that oxygen radicals have a key role in cardiac injury. ${ }^{38,39}$ Studies showed that ISO-induced cardiotoxicity was partly due to the formation of oxygen-free radicals and sulfhydryl reactivity through a variety of its oxidation products. ${ }^{2}$ Cells are protected against oxidative insult by diverse antioxidant enzymes such as SOD and GSH-Px. ${ }^{40}$ SOD catalyzes the dismutation of $\mathrm{O}_{2}^{--}$to $\mathrm{H}_{2} \mathrm{O}_{2}$ in the extracellular 
compartment. The major pathways for the disposal of $\mathrm{H}_{2} \mathrm{O}_{2}$ in cells are catalyzed by GSH-Px and catalase, which metabolize $\mathrm{H}_{2} \mathrm{O}_{2}$ into water and oxygen. Du and coworkers ${ }^{41}$ reported that endogenous $\mathrm{SO}_{2}$ raised the antioxidant capacity in rats of monocrotaline-induced pulmonary hypertension. In this study, antioxidant activity demonstrated by SOD and GSH-Px in the myocardial tissue of ISO-treated rats decreased significantly. mRNAs of SOD1, SOD2, and GSH$P \times 1$ were significantly lower than those of the control group, but $\mathrm{O}_{2}^{\bullet-}$ and $\mathrm{H}_{2} \mathrm{O}_{2}$ increased significantly compared with control rats. After supplementation with exogenous donors of $\mathrm{SO}_{2}$, the activity of SOD and GSH-Px increased, SOD2 and GSH-Pxl mRNAs were also upregulated, whereas the content of $\mathrm{O}_{2}^{--}$and $\mathrm{H}_{2} \mathrm{O}_{2}$ decreased, indicating that $\mathrm{SO}_{2}$ could improve the antioxidant capacity of myocardium and reduce free radicals production. Meanwhile, supplemental $\mathrm{SO}_{2}$ could ameliorate cardiac function injured by ISO. The results suggested that $\mathrm{SO}_{2}$ could alleviate myocardial damage induced by ISO via increasing antioxidant capacity. The study also showed that ISO caused a downregulation of endogenous $\mathrm{SO}_{2}$ /GOT pathway. Taken together, the above findings suggested that the disturbed endogenous $\mathrm{SO}_{2} / \mathrm{GOT}$ pathway is associated with the imbalance of antioxidant/ oxidant capacities generated by ISO.

Taken together, these results indicated that the downregulation of the endogenous $\mathrm{SO}_{2} / \mathrm{GOT}$ pathway is probably involved with the pathogenesis of ISO-induced myocardial injury. $\mathrm{SO}_{2}$ improved ISO-induced myocardial damage and cardiac function in association with increasing myocardial antioxidant capacity.

\section{ACKNOWLEDGEMENTS}

This work was supported by the National Natural Science Foundation of China (30630031, 30821001 and 30801251), Key Program of Science and Technology, Ministry of Education, China (307001), and the Major Basic Research Program of China (2006CB503807).

\section{DISCLOSURE/CONFLICT OF INTEREST}

The authors declare no conflict of interest.

1. Rona G. Catecholamine cardiotoxicity. J Mol Cell Cardiol 1985;17: 291-306.

2. Singal PK, Yates JC, Beamish RE, et al. Influence of reducing agents on adrenochrome-induced changes in the heart. Arch Pathol Lab Med 1981;105:664-669.

3. Noronha-Dutra AA, Steen-Dutra EM, Woolf N. Epinephrine-induced cytotoxicity of rat plasma. Its effects on isolated cardiac myocytes. Lab Invest 1988;59:817-823.

4. Rich DQ, Schwartz J, Mittleman MA, et al. Association of short term ambient air pollution concentrations and ventricular arrhythmias. Am J Epidemiol 2005;161:1123-1132.

5. Park SK, O'Neill MS, Vokonas PS, et al. Effects of air pollution on heart rate variability: the VA normative aging study. Environ Health Perspect 2005;113:304-309.

6. D'Ippoliti D, Forastiere F, Ancona C, et al. Air pollution and myocardial infarction in Rome: a case-crossover analysis. Epidemiology 2003; 14:528-535.

7. Du SX, Jin HF, Bu DF, et al. Endogenously generated sulfur dioxide and its vasorelaxant effect in rats. Acta Pharmacol Sin 2008;29:923-930.

8. Mitsuhashi H, Ota F, Ikeuchi K, et al. Sulfite is generated from PAPS by activated neutrophils. Tohoku J Exp Med 2002;198:125-132.
9. Ji AJ, Savon SR, Jacobsen DW. Determination of total serum sulfite by HPLC with fluorescence detection. Clin Chem 1995;41:897-903.

10. Balazy M, Abu-Yousef IA, Harpp DN, et al. Identification of carbonyl sulfide and sulfur dioxide in porcine coronary artery by gas chromatography/mass spectrometry, possible relevance to EDHF. Biochem Biophys Res Commun 2003;311:728-734.

11. Zhang SQ, Du JB, Jin HF, et al. Endogenous sulfur dioxide aggravates myocardial injury in isolated rat heart with ischemia and reperfusion. Transplantation 2009;87:517-524.

12. Beck-Speier I, Dayal N, Maier KL. Pro-inflammatory response of alveolar macrophages induced by sulphite: studies with lucigenin-dependent chemiluminescence. J Biolumin Chemilumin 1998;13:91-99.

13. Kaye AD, De Witt BJ, Anwar M, et al. Analysis of responses of garlic derivatives in the pulmonary vascular bed of the rat. J Appl Physiol 2000;89:353-358.

14. Meng $\mathrm{Z}$, Geng $\mathrm{H}$, Bai J, et al. Blood pressure of rats lowered by sulfur dioxide and its derivatives. Inhal Toxicol 2003;15:951-959.

15. Rona $\mathrm{G}$, Chappel $\mathrm{Cl}$, Balazs $\mathrm{T}$, et al. An infarct-like myocardial lesion and other toxic manifestations produced by isoproterenol in the rat. AMA Arch Pathol 1959;67:443-455.

16. Zhao X, Jin HF, Du SX, et al. The effect of sulfur dioxide on blood pressure and aortic structure of spontaneously hypertensive rat. Chin Pharmacol Bull 2008;24:327-330.

17. Weibel ER. Stereological principles for morphometry in electron microscopic cytology. Int Rev Cytol 1969;26:235-302.

18. Romek M., Krzysztofowicz E.. Stereological analysis of mitochondria in embryos of Rana temporaria and Bufo bufo during cleavage. Folia Histochem Cytobiol 2005;43:57-63.

19. Gong L, Wang ZG, Ran HT, et al. Relationship between myocardial ultrasonic integrated backscatter and mitochondria of the myocardium in dogs. Clin Imaging 2006;30:402-408.

20. Ubuka T, Yuasa S, Ohta J, et al. Formation of sulfate from L-cysteine in rat liver mitochondria. Acta Med Okayama 1990;44:55-64.

21. Mitsuhashi $H$, Ikeuchi $H$, Yamashita $S$, et al. Increased levels of serum sulfite in patients with acute pneumonia. Shock 2004;21: 99-102.

22. Yang $X$, Zhao $Y$, Zhou $Y$, et al. Component and antioxidant properties of polysaccharide fractions isolated from Angelica sinensis (OLIV) DIELS. Biol Pharm Bull 2007;30:1884-1890.

23. Csiszar A, Ungvari Z, Edwards JG, et al. Aging-induced phenotypic changes and oxidative stressimpair coronary arteriolar function. Circ Res 2002;90:1159-1166.

24. Ungvari Z, Csiszar A, Edwards JG, et al. Increased superoxide production in coronary arteries inhyperhomocysteinemia: role of tumor necrosis factor-alpha, NAD(P)Hoxidase, and inducible nitric oxide synthase. Arterioscler Thromb Vasc Biol 2003;23:418-424.

25. Ungvari Z, Csiszar A, Huang A, et al. High pressure induces superoxide production in isolated arteries viaprotein kinase Cdependent activation of $\mathrm{NAD}(\mathrm{P}) \mathrm{H}$ oxidase. Circulation 2003;108: 1253-1258.

26. Miura H, Bosnjak JJ, Ning G, et al. Role for hydrogen peroxide in flowinduced dilation of human coronaryarterioles. Circ Res 2003;92: e31-e40.

27. Brilla CG, Maisch B, Rupp $\mathrm{H}$, et al. Pharmacological modulation of cardiac fibroblast function. Herz 1995;20:127-134.

28. Todd GL, Baroldi G, Pieper GM, et al. Experimental catecholamineinduced myocardial necrosis I. Morphology, quantification and regional distribution of acute contraction band lesions. J Mol Cell Cardiol 1985;17:317-338.

29. Jalil JE, Doering CW, Janicki JS, et al. Fibrillar collagen and myocardial stiffness in the intact hypertrophied rat left ventricle. Circ Res 1989;64:1041-1050.

30. Benjamin IJ, Jalil JE, Tan LB, et al. Isoproterenol-induced myocardial fibrosis in relation to myocyte necrosis. Circ Res 1989;65:657-670.

31. Grimm D, Elsner D, Schunkert $H$, et al. Development of heart failure following isoproterenol administration in the rat: role of the renninangiotensin system. Cardiovasc Res 1998;37:91-100.

32. Stipanuk MH. Metabolism of sulfur-containing amino acids. Ann Rev Nutr 1986;6:179-209.

33. Griffith OW. Cysteinesulfinate metabolism, altered partitioning between transamination and decarboxylation following administration of beta-methylene aspartate. J Biol Chem 1983;258: 1591-1598. 
34. Rathore $\mathrm{N}$, John $\mathrm{S}$, Kale $\mathrm{M}$, et al. Lipid peroxidation and antioxidant enzymes in isoproterenol induced oxidative stress in rat tissues. Pharmacol Res 1998;38:297-303.

35. Meszaros J, Levai G. Ultrastructural and electrophysiological alterations during the development of catecholamine-induced cardiac hypertrophy and failure. Acta Biol Hung 1990;41:289-307.

36. Neri M, Cerretani D, Fiaschi Al, et al. Correlation between cardiac oxidative stress and myocardial pathology due to acute and chronic norepinephrine administration in rats. J Cell Mol Med 2007;11: 156-170.

37. Mohanty I, Arya DS, Dinda A, et al. Mechanisms of cardioprotective effect of Withania somnifera in experimentally induced myocardial infarction. Basic Clin Pharmacol Toxicol 2004;94:184-190.
38. Akhlaghi $\mathrm{M}$, Bandy $\mathrm{B}$. Mechanisms of flavonoid protection against myocardial ischemia-reperfusion injury. J Mol Cell Cardiol 2009; 46:309-317.

39. Kim HJ, Tsoy I, Park JM, et al. Anthocyanins from soybean seed coat inhibit the expression of TNF-alpha-induced genes associated with ischemia/reperfusion in endothelial cell by NF-kappaB dependent pathway and reduce rat myocardial damages incurred by ischemiaand reperfusion in vivo. FEBS Lett 2006;580:1391-1397.

40. Iskesen I, Saribulbul O, Cerrahoglu M, et al. Trimetazidine reduces oxidative stress in cardiac surgery. Circ J 2006;70:1169-1173.

41. Jin HF, Du SX, Zhao X, et al. Effects of endogenous sulfur dioxide on monocrotaline-induced pulmonary hypertension in rats. Acta Pharmacol Sin 2008;29:1157-1166. 